\title{
Phonon Spectra of Disordered Chains: An Application to DNA
}

\author{
S. C. Gómez-Carrillo ${ }^{1}$, K. M. Fonseca ${ }^{1}$, and R. R. Rey-González ${ }^{1}$ \\ 1 Departamento de Física, Universidad Nacional de Colombia, Ciudad Universitaria, Bogotá, Colombia
}

Received on 8 December, 2005

\begin{abstract}
Recent developments in the field of nano-electronics have encouraged the study of quasi one dimensional systems such as DNA and its applications to new devices. In this work we use two models to explore the vibrational properties of DNA-like chains: a linear chain and two laterally coupled linear chains. In the former case, the disorder induces a diatomic behavior, while monoatomic characteristics are displayed in the last case.
\end{abstract}

Keywords: Disordered chains; DNA

\section{INTRODUCTION}

Increasing drive for miniaturization and improved performance in electronic devices, and potential applications in the nanotechnologies has thrust forward an impressive growth of research effort to investigate the properties of novel nanowire materials. It is foreseen that conventional solid-state technology could be replaced by new generations of devices based on molecular components, which take advantage of quantum mechanical effects of few particles and/or excitations. In this scenario DNA molecules seem to be particulary suitable due to their flexibility, self-assembling properties and their well structured and reproducible architectures $[1,2]$. However, one should be aware that at the moment there are contradictory experimental results about whether DNA is an insulator, a semiconductor, a metal, or a superconductor [3-5].

DNA vibrational properties have been investigated over the last 20 years using Raman and IR spectroscopy. While the understanding of fine structure details in Raman spectroscopy needs a complete description on the atomic level, it is possible to use simple mechanical models to understand macromolecular motion, including bending, twisting and stretching. In particular, if an analysis of the effects of hydrogen bonds is desired, single-chain models are not enough and doble-chain models are in order [6]. As in several models discussed in the literature over the last 20 years [7-9], we describe the DNA molecule as a single or a double chain, where the nucleotides are represented by effective sites, connected to each other by means of effective elastic constants. The double chain model allows us to analyze the role of interchain coupling in the vibrational properties. Finally, since DNA is not a periodic system, disorder effects -which determine key features of DNA beavior- are introduced in the models.

\section{MODEL AND THEORY}

The number of atoms and the masses of the building blocks of DNA, sugar, phosphate and the four fundamental bases: Adenine (A), Thymine (T), Cytosine (C) and Guanine (G) [10, 11], are shown in table II. Chargaff and collaborators [12] found that bases, bounded by hydrogen brides, always appear in pairs: Adenine with Thymine (A-T pair) and Cytosine with Guanine (C-G pair).

\begin{tabular}{|c|c|c|c|c|c|c|}
\hline Base or unit & $\mathrm{O}$ & $\mathrm{N}$ & $\mathrm{C}$ & $\mathrm{P}$ & $\mathrm{H}$ & Total mass (a.m.u.) \\
\hline Adenine & 0 & 5 & 5 & 0 & 4 & 134.1226 \\
\hline Thymine & 2 & 2 & 5 & 0 & 5 & 125.1104 \\
\hline Cytosine & 1 & 3 & 4 & 0 & 4 & 110.0977 \\
\hline Guanine & 1 & 5 & 5 & 0 & 4 & 150.1226 \\
\hline Sugar & 1 & 0 & 5 & 0 & 7 & 83.1128 \\
\hline Phosphate & 4 & 0 & 0 & 1 & 1 & 95.9817 \\
\hline
\end{tabular}

TABLE I: Number of atoms (O, oxygen; N, nitrogen; C, carbon; $\mathrm{P}$, phosphate; $\mathrm{H}$, hydrogen) and mass for every base.

\section{A. Linear chain model}

As a first approximation, we model DNA as an one dimensional linear chain with masses which take the values $m_{1}, m_{2}$ and $m_{p}$, where $m_{1}$ represents the A-T pair plus two sugar units, $m_{2}$ represents the C-G pair plus two sugar units and $m_{p}$ is formed by two phosphates. These masses are calculated in table II and the respective model is depicted in Fig. 1. We can observe high similarity in the masses $m_{1}$ and $m_{2}$.

\begin{tabular}{|c|c|c|c|c|c|c|}
\hline Base or unit & $\mathrm{O}$ & $\mathrm{N}$ & $\mathrm{C}$ & $\mathrm{P}$ & $\mathrm{H}$ & Total mass (a.m.u.) \\
\hline$m_{1}$ & 4 & 7 & 20 & 0 & 23 & 425.4586 \\
\hline$m_{2}$ & 4 & 8 & 19 & 0 & 22 & 426.4459 \\
\hline$m_{p}$ & 8 & 0 & 0 & 2 & 2 & 191.9634 \\
\hline
\end{tabular}

TABLE II: As table I for $m_{1}, m_{2}$ and $m_{p}$ masses.

We begin by investigating a periodic chain, in which the unit cell, with $a_{0}$ lattice parameter, comprises four masses, two $m_{p}$ masses and one $m_{1}$ and one $m_{2}$ mass. In order to model the elastic forces among them we assume the linear regime and use two different constant forces $k_{1}=$ 0.090 mdynes $/ \AA$ and $k_{2}=0.075$ mdynes $/ \AA$ [7]. Later on we introduce disorder such that $m_{p}$ masses stay put on their sites, while the masses $m_{1}\left(m_{2}\right)$ occupy half of the remaining places.

The hamiltonian of the linear chain is

$$
\widehat{H}=\sum_{i} \frac{1}{2} M_{i} \dot{u}_{i}^{2}+\sum_{i} \frac{1}{2} K_{i}\left(u_{i+1}-u_{i}\right)^{2},
$$

where $M_{i}$ is the $i$-th mass, $K_{i}$ the coupling constant between masses $i$ and $i+1$, and $u_{i}$ is the displacement of the $i$-th mass 


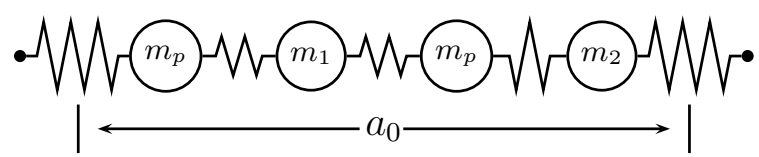

FIG. 1: Unitary cell for one dimensional chain

with respect to its equilibrium position. Using Bloch's theorem we search the solutions of the dynamical equations generated by the Hamiltonian (1) employing the Ansatz

$$
u_{i}(q, t)=\frac{v_{[i]}(q)}{\sqrt{M_{i}}} e^{i\left(q a_{0}-\omega t\right)},
$$

where, owing to the periodicity of the lattice there are only four different amplitudes $v_{[i]}(q)$. The notation $[i]$ stands for index $i$ module 4 , and $q$ denotes the wavevector. Replacing (2) into the equations of motion we find

$$
\left(\omega^{2} \mathbb{I}-\mathbb{D}(q)\right)|\vec{v}(q)\rangle=0,
$$

where $\mathbb{I}$ designates the identity matrix. The matrix $\mathbb{D}(q)$, known as the dynamical matrix [13, 14], is given by

$$
\left(\begin{array}{cccc}
\omega^{2}-\frac{k_{1}+k_{2}}{m_{p}} & \frac{k_{1}}{\sqrt{m_{p} m_{1}}} & 0 & \frac{\mathrm{e}^{-i q a} k_{1}}{\sqrt{m_{p} m_{2}}} \\
\frac{k_{1}}{\sqrt{m_{p} m_{1}}} & \omega^{2}-\frac{2 k_{1}}{m_{1}} & \frac{k_{1}}{\sqrt{m_{p} m_{1}}} & 0 \\
0 & \frac{k_{1}}{\sqrt{m_{p} m_{1}}} & \omega^{2}-\frac{k_{1}+k_{2}}{m_{p}} & \frac{k_{2}}{\sqrt{m_{p} m_{2}}} \\
\frac{k_{1} \mathrm{e}^{i q a}}{\sqrt{m_{p} m_{2}}} & 0 & \frac{k_{2}}{\sqrt{m_{p} m_{2}}} & \omega^{2}-\frac{k_{2}}{m_{2}}
\end{array}\right) .
$$

With the purpose to analyze disordered chains is convenient to calculate the density of states (DOS). We can do it by means of Green's function, $G(z)$, which is defined as [15]:

$$
G(z)=\int d \omega \frac{|\vec{v}(q(\omega))><\vec{v}(q(\omega))|}{z-\omega}
$$

where the integration extends over all of the eigenvalues of (3). Here, $z$ is a complex variable whose real part designates the energy of the system and whose imaginary part is an infinitesimal term to avoid the discontinuity that is obtained in the complex plane. The DOS is defined as the imaginary part of the trace of Green's function,

$$
\mathrm{D}(\omega)=\lim _{\varepsilon \rightarrow 0} \frac{1}{\pi} \operatorname{Im}(\operatorname{Tr} G(\omega+i \varepsilon)) .
$$

Figures 2 and 3 show the dispersion relation and the DOS for the ordered one-dimensional chain. In both situations we observe four branches: three optical and one acoustical branch. We see that the gap between the acoustical branch and the first optical branch is very narrow.

In the Fig. II B we have plotted the dispersion relations for diatomic chains formed by $m_{p}$ and $m_{1}$ (A-T pair) masses or $m_{p}$ and $m_{2}$ (C-G pair) masses. It is clear that $\mathrm{C}-\mathrm{G}$ pair provides the highest optical frequencies.

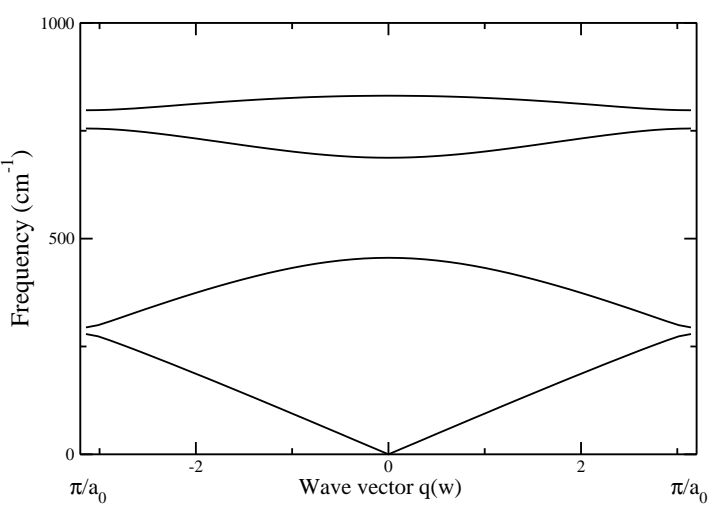

FIG. 2: Dispersion relation for one dimensional chain.

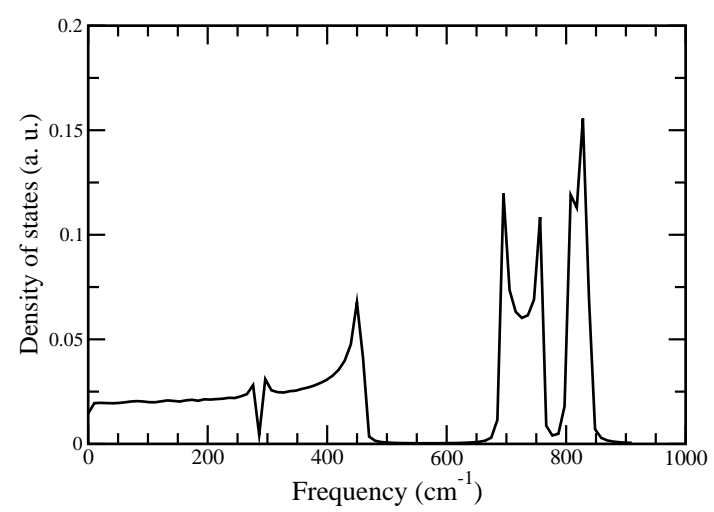

FIG. 3: DOS for one dimensional chain

\section{B. Double chain model}

With the purpose to model the DNA in more realistic form, we use a periodic double chain model. This model is composed by four masses, $m_{1}, m_{2}, m_{3}$ and $m_{4}$. Every mass corresponds to a base plus sugar and phosphate units. For the most simple situation the unit cell has four masses, as the model of the previous section but with a larger number of elastic constants.

Figure 5 is a schematic representation for this case. This model permit us to have very complex unit cell that containing more than 20 masses, when we take into account the different configurations with the bases pairs.

If we compare Fig. 6, which shows the dispersion relation for the most simple case of the double chain model, with Fig. 2, we observe four branches in both cases, but with a different behavior. Contrasting the corresponding DOS, depicted in Figs. 7 and 3, respectively, we appreciate more clearly the difference. The DOS for the double chain model exhibits a more complex structure with smaller gaps. 


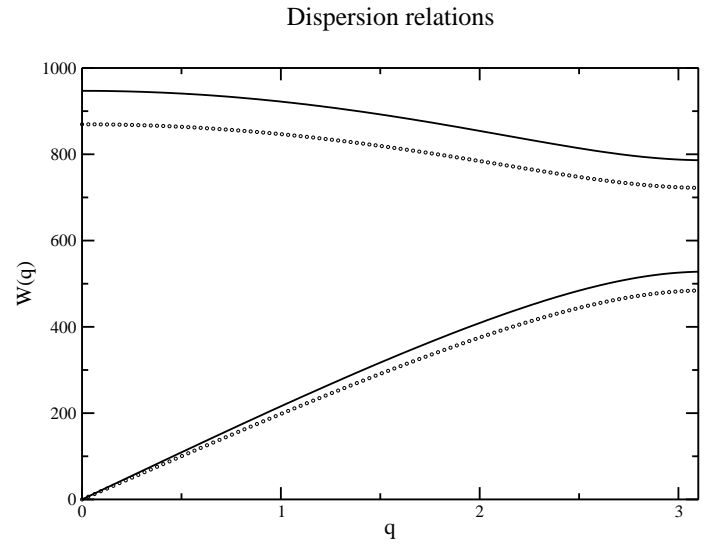

FIG. 4: Relation of dispersion for 1D linear chains. A-T pair (continuous line) and $\mathrm{C}-\mathrm{G}$ (circles).

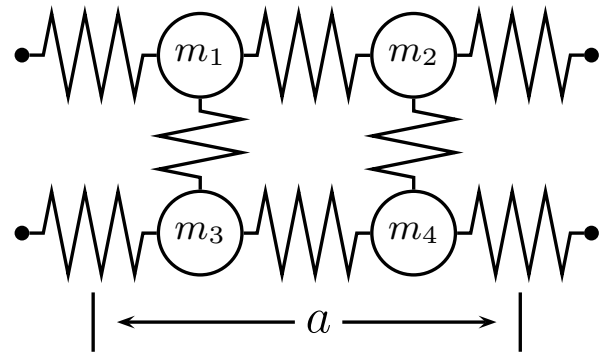

FIG. 5: Unit cell for the double chain model.

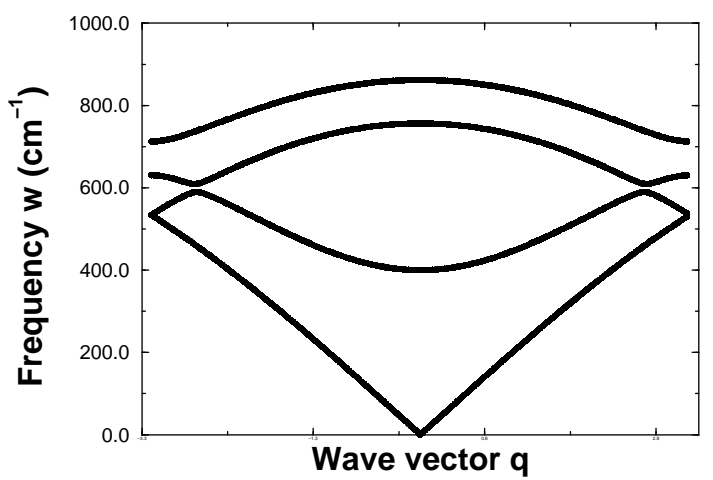

FIG. 6: Dispersion relation for double chain model.

The rich structures of the dispersion relation and the DOS of the most complex double chain model, i.e., when we take into account all the posible configurations for the pairs, but with only A-T and C-G bounds, are shown in Figs. 8 and III.

\section{DISORDERED MODELS}

Taking into account that the difference of genetic code is given by the disorder of DNA bases, we study the behavior of

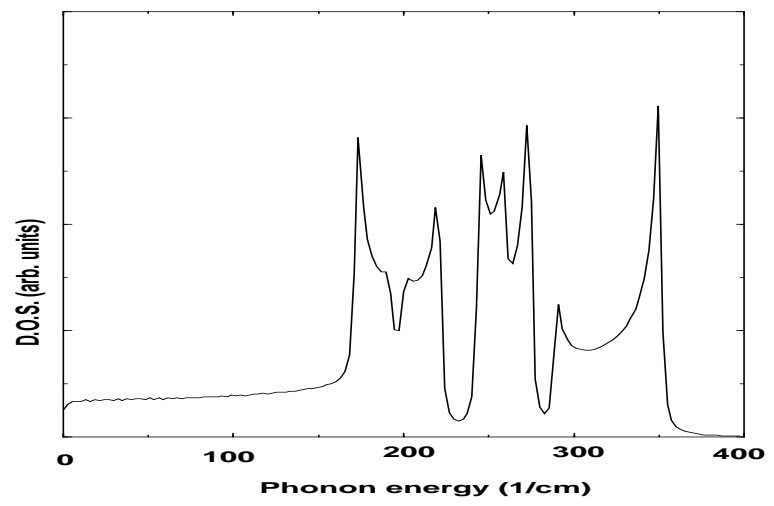

FIG. 7: DOS for double chain model.

the density of states for disordered chains. We enforce one important characteristic of DNA chains, in which phosphates always occupy a place among base-sugar groups placing a mass $m_{p}$ between $m_{1}$ or $m_{2}$ masses. The probabilities of masses $m_{1}$ or $m_{2}$, whose positions are random, are equal respecting the experimental finding.

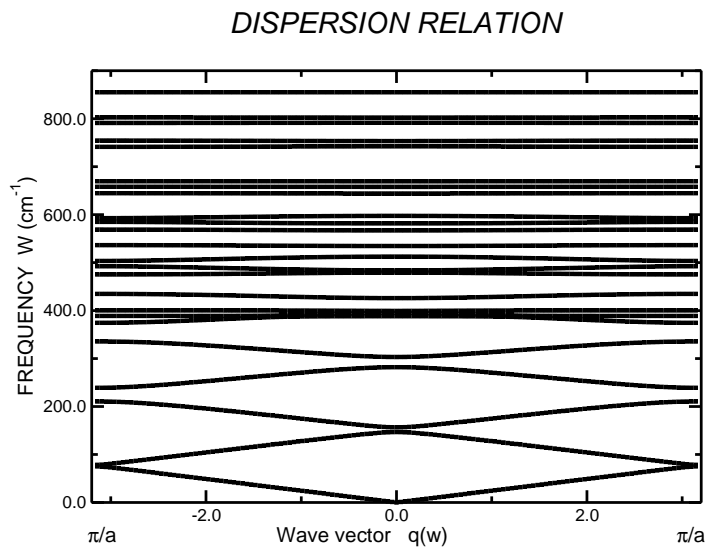

FIG. 8: Dispersion relation for the most complex periodic double chain model

The DOS for the disordered linear chain model, Fig. 10, shows the suppression of two small gaps and the marked presence of only two branches. The absence or presence of (interface) states in the gap depend on the type of masses at the chain ends, as a careful analysis reveals. In contrast, for the disordered double-chain model, the DOS displayed in Fig. 11 does not show any gaps.

However, it is possible to identify a light envelop form resembling the acoustical and optical branches. Results, not shown here, permit us to associate these structures with double chains formed by $\operatorname{Poly}(\mathrm{C})$-chains (Poly(A)-chains) laterally coupled with $\operatorname{Poly}(\mathrm{G})$-chains (Poly(T)-chains). 


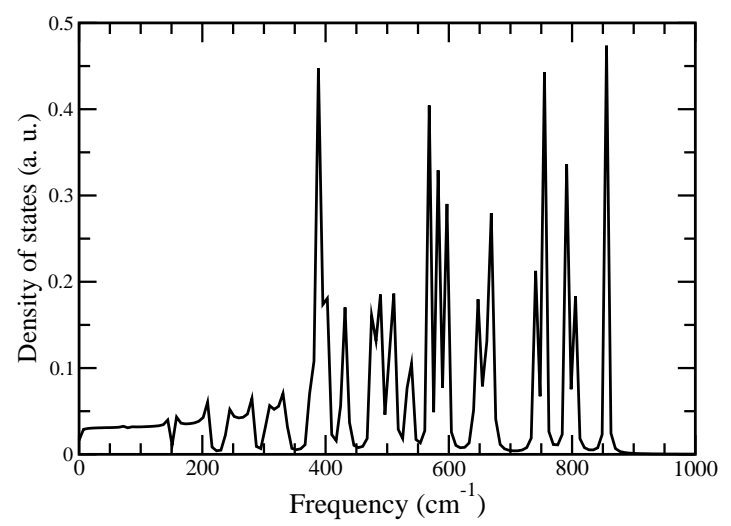

FIG. 9: DOS for the most complex periodic double chain model

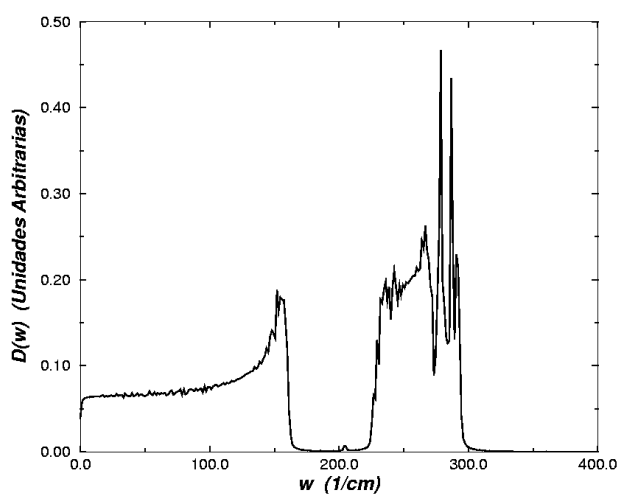

FIG. 10: DOS for a disordered linear chain model.

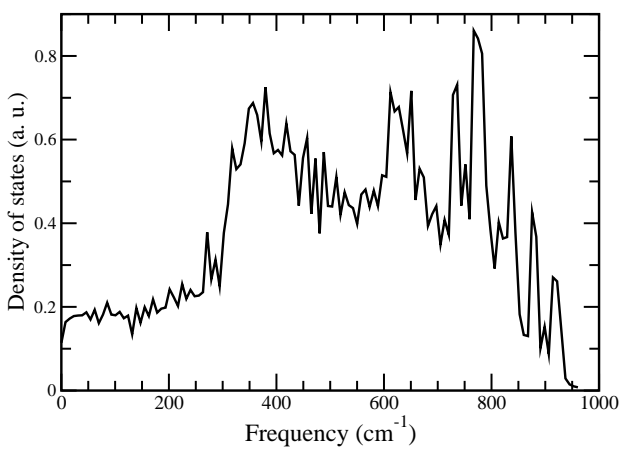

FIG. 11: DOS for a disordered linear double-chain model.

\section{CONCLUSIONS}

We model DNA-like chains, in two different ways. Notwithstanding their simplicity, these models allow for an understanding of some vibrational properties of this sort of systems. Disordered DNA-like linear chains present a diatomic behavior, in spite of being composed by at least three different masses. On the other hand, introducing lateral correlations leads to even a more simple behavior: although the disordered double-chains model presented here has a very complex unit cell it exhibits monatomic characteristics.

\section{Acknowledgments}

The authors would like to acknowledge financial support from Colciencias as well from Universidad Nacional, Colombia.
[1] A. Yu Kasumov, D. V. Klinov, P. E. Roche, S. Gueron, and H. Bouchiat, Appl. Phys. Lett. 84, 1007 (2004).

[2] http://www.cnrs.fr/Cnrspresse/n391/html/en391a02.htm, march 2001.

[3] D. Porath, A. Bezryadin, S. de Vries, and C. Dekker, Nature, 403, 635 (2000).

[4] A. J. Storm, J. van Noort, S. de Vries, and C. Dekker, Appl. Phys. Lett. 79, 23 (2001).

[5] Hans-Werner Fink and Christian Schönenberger, Nature, 398, 407 (1999).

[6] S. C. Gómez-Carrillo, P. A. Schulz, and R. R. Rey-González, Unpublished.

[7] J. W. Powell, G. S. Edwards, L. Genzel, F. Kremer, and A. Wittlin, Phys. Rev. A. 35, 3929 (1987).

[8] L. L. Van Zandt and V. K. Saxena, Phys. Rev. A. 35, 2672
(1989).

[9] A. Wittlin, L. Genzel, F. Kremer, S. Häseler, and A. Poglitsch, Phys. Rev. A. 34, 493 (1986).

[10] L. Pauling and R. P. Corey, Nature, 171, 346 (1953).

[11] J. D. Watson and F. H. Crick, Nature, 171, 737 (1953).

[12] S. Zamenhof, G. Brawerman, and E. Chargaff, Biochem. and Biophys. Acta 9, 402 (1952).

[13] A. A. Maradudin, E. W. Montroll, and G. H. Weiss, Theory of lattice dynamics in the harmonic approximation, Academic Press Inc., 1963.

[14] Gerald Burns, Solid State Physics, Academic Press, Inc, 1985.

[15] E. N. Economou, Greens Functions in Quantum Physics, Springer-Verlag, 1983. 